\title{
LAS MUJERES JÓVENES EN ESPAÑA. (NUEVAS) PRECARIEDADES Y (VIEJAS) DESIGUALDADES
}

Mercedes Alcañiz, Vicent Querol e Ana Marti

Universitat Jaume I. Castellón, España

\section{Resumen}

La crisis económica y el incremento de la precariedad en el mercado laboral producida por el contexto de neo-liberalismo, desregulación y globalización en el que nos hallamos, ha afectado mayormente a la población joven en la Unión Europea. El artículo analiza la situación de precariedad de las mujeres jóvenes en el mercado laboral así como su desigualdad respecto a los jóvenes varones y la percepción que tienen dichas jóvenes respecto a si su situación es desigual a la de sus compañeros. La metodología empleada ha consistido en la explotación de datos secundarios obtenidos de fuentes oficiales españolas y europeas y en el análisis de los discursos de 24 mujeres y hombres comprendidos entre los 16 y los 34 años en España.

Palavras-clave: juventud, precariedad, género, desigualdad, percepción.

\section{Resumo}

As mulheres jovens em espanha. (Novas) precariedades e (velhas) desigualdades

A crise económica e o aumento da precariedade no mercado de trabalho produzida pelo contexto de neoliberalismo, desregulação e globalização no qual nos encontramos, tem afectado maioritariamente a população jovem na União Europeia. $\mathrm{O}$ artigo analisa a situação de precariedade das mulheres jovens no mercado de trabalho assim como a desigualdade em relação aos jovens do sexo masculino e a percepção que têm as jovens acerca de se a sua situação é desigual em relação à dos seus companheiros. A metodologia utilizada consistiu na exploração de dados secundários, obtidos de fontes oficiais espanholas e europeias, e a análise dos discursos de 24 pessoas, mulheres e homens com idades compreendidas entre os 16 e os 24 anos, em Espanha.

Palavras-chave: juventude, precariedade, género, desigualdade, percepção.

\section{Abstract}

Young women in Spain. (New) precariousness and (old) inequalities

The economic crisis and increased precariousness in the labour market resulting from the current context of neoliberalism, deregulation and globalisation have mostly affected the young population of the European Union. The article analyses the situation of labour market precariousness faced by young women and their inequality with young men, to get to know if such young women perceive their situation to be unequal to that of their male counterparts. The methods used in each case have been, respectively, the exploitation of secondary data obtained from official Spanish and European sources, and the analysis of the discourses of 24 women and men aged between 16 and 34 years living in Spain.

Keywords: youth, precariousness, gender, inequality, perception. 


\section{Introducción}

En 2007-2008 se originó en los Estados Unidos la denominada gran recesión o crisis financiera (Calvo, 2009; Estefanía, 2011; Stiglitz, 2002 y 2010) extendida posteriormente a los países europeos, y cuya manifestación en el ámbito laboral ha sido la aprobación de diversas normativas ${ }^{1}$ cuyo objetivo era la población laboral y en concreto la extensión de una mayor flexibilidad y desregulación; acontecimiento vaticinado con anterioridad por Robert Castel (1999) como «el cuestionamiento de la estabilidad vinculada a la condición laboral». Este contexto de crisis económica ha afectado de manera especial a los colectivos más vulnerables - las mujeres, los y las jóvenes, la población inmigrante y las personas con poca cualificación y desempleadas de larga duración - los cuales quedan en situación de mayor precariedad.

Por precariedad (Alonso y Fernández, 2013; Bourdieu, 1999; Castel, 1999; Foessa, 2014; Standing, 2013) se alude a las condiciones de inseguridad, pérdida de derechos sociales, inestabilidad e inseguridad y bajos salarios que caracterizan a muchos empleos en la actualidad y que ya se considera un rasgo de la estructura social actual (Foessa, 2014). El perfil de precario/a sería el de una persona con un trabajo temporal, o con una jornada a tiempo parcial, o sub-contratado, o sin contrato, con bajo salario, escasa protección social y sin probabilidades de promoción (Standing, 2013). Esta situación en el mercado laboral se ha incrementado en los últimos años y afecta sobre todo a los colectivos «más nuevos», como los y las jóvenes, las mujeres y los y las inmigrantes.

La problemática de la incorporación de la juventud al mercado laboral, considerada, junto con la emancipación, como las variables identificadoras del proceso de transición a la vida adulta, ha sido resaltado por la literatura sobre estudios de la juventud desde los años 80 y sobre todo desde 2008 (Benedicto et al, 2014; Bradley y Devadason, 2008; Casal et al, 2006 y 2011; Ferreira y Nunes, 2013; Guerreiro y Abrantes, 2005; Moreno, 2012; Pais, 2002). Ahora bien, la población joven se incorpora al mercado laboral desde posiciones diferentes según su sexo, su clase social, su formación, su etnia, su hábitat, etc (Furlong y Cartmel, 1997). En nuestro caso, nos preguntamos si las consecuencias de la crisis divergen según el sexo y si las mujeres jóvenes tienen una mayor precariedad laboral debido a que están afectadas por unas circunstancias de doble desigualdad, por ser mujeres y por ser jóvenes, y ello, a pesar de la similar o superior presencia de las mujeres en el sistema educativo.

1 En el caso español, la última reforma laboral aprobada ha sido el Real Decreto-Ley 3/2012 de 10 de febrero de Medidas urgentes para la reforma del mercado laboral, normativa que refleja el actual proceso de flexibilización apoyado por el estado (Casaca, 2012; Kovacs, 2005; Oliveira, 2011; Sennett, 2001) y que permite la realización de nuevas jornadas y horarios adecuados a las exigencias productivas actuales relacionadas con la competitividad en la economía global. 
La intersección ${ }^{2}$ entre dos aspectos de desigualdad en el colectivo de mujeres jóvenes alude, en primer lugar, el centrado en la precariedad laboral y, en segundo lugar, el referido a la desigualdad de género, considerando que dada la tradicional fragilidad y vulnerabilidad de las mujeres en el mercado laboral (Instituto de la Mujer, 2006; SEPE, 2014), la transición de las mujeres jóvenes a la vida adulta puede resultar más difícil que para los hombres (Asián-Chaves y Rodríguez, 2014; Plantenga, 2013) debido a la existencia de discriminación en el mercado laboral y a la continuidad en el reparto de los roles domésticos y de cuidado entre mujeres y hombres (Torns y Recio, 2012).

Este último aspecto, refuerza el proceso de precariedad y de desigualdad de las mujeres jóvenes, determinando, en la mayoría de los casos el cómo, el donde y el cuándo del desempeño de la actividad laboral (Albert, Toharia y Davia, 2008; Alcañiz, 2013; Cano, 2000; Casaca, 2011; Ferreira y Monteiro, 2013) y, el que, pese al incremento de mujeres ocupadas, éstas siguen siendo, y se siguen percibiendo, como las principales responsables de las tareas domésticas y de cuidado; manifestación evidente de la nueva lógica neo-patriarcal (Webb, 2010) en alusión a que la flexibilización de la jornada laboral femenina se considera una forma de compatibilizar el trabajo productivo y el trabajo reproductivo (Campillo, 2010; Stratigaki, 2004; Torns, 2007 y 2011).

Por ello, el objetivo del presente artículo es doble: en primer lugar, analizar la situación de las mujeres jóvenes en el mercado laboral así como su desempeño en el ámbito doméstico en España y, en segundo lugar, conocer la percepción que tienen sobre si su situación es desigual respecto a la de sus compañeros e compañeras. Este doble objetivo, facilita la visibilidad, por una parte, de la situación real, objetiva, de las mujeres jóvenes respecto al trabajo remunerado y el de cuidados así como su relación con la precariedad y la desigualdad y, por otra, de la visión subjetiva que aprecian ellas sobre su situación respecto a la desigualdad de género.

El artículo lo estructuramos de la siguiente manera: en primer lugar describimos la doble metodología utilizada en la investigación; en segundo lugar, nos centramos en la exposición de los resultados centrados en la situación de las mujeres jóvenes en el mercado laboral y en el análisis de las percepciones de dichas mujeres sobre su situación de desigualdad respecto de los hombres y, en último lugar, se exponen las conclusiones. Al final del texto se incluye una tabla con los perfiles de los y las entrevistadas.

La teoría de la interseccionalidad, propone y examina cómo diferentes categorías de discriminación, construidas social y culturalmente, interactúan en múltiples niveles, contribuyendo con ello a reforzar la desigualdad social (Yuval-Davis, 2006), 


\section{Metodología}

El objeto de estudio del presente artículo se centra en la juventud española, fundamentalmente en lo que atañe a las mujeres jóvenes. Ello nos exige delimitar conceptualmente el significado de «juventud» (Brunet y Prizzi, 2013; Casal et al, 2006 y 2011; Furlong, 1997; Furlong, 2013; País, 2002) y, de manera especial, los límites de edad que marcan a esta categoría social (Erikson, 1980; Feixas, 1998, 2001 y 2014; Furlong, 2013; Leccardi, 2005).

La mayoría de la literatura académica y oficial actual consultada, referencia como «juventud» a los grupos de edad comprendidos entre los 16 y los 29 años. En algunos casos, esta delimitación se alarga hasta los 34 años debido a las dificultades encontradas por los y las jóvenes en acceder al mercado laboral estable y consecuentemente a una emancipación que suponga la creación de un hogar independiente.

La metodología utilizada ha sido la siguiente: en relación con el primer objetivo, se ha recurrido a la consulta de fuentes secundarias fundamentalmente provenientes de organismos oficiales españoles y de la oficina estadística europea, EUROSTAT. Respecto a las fuentes españolas se ha consultado: en el Instituto Nacional de Estadística (INE), la Encuesta de Población Activa (EPA), los Censos de Población y viviendas, la Encuesta de Estructura Salarial; en el INJUVE, los sondeos de Opinión de 2001 y 2008 así como publicaciones y estudios relacionados con el tema estudiado; en el Centro de Investigaciones Sociológicas (CIS), en concreto los Estudios no 2578 y 2911. Respecto a EUROSTAT, se ha consultado la información proporcionada por la Labour Force Survey (LFSs), Youth on the Move así como la European Quality of Life Survey (EQLS).

En relación con el segundo objetivo - conocer la percepción que las jóvenes tienen sobre su situación - recurrimos a la investigación cualitativa realizada por nuestro equipo «El impacto de la crisis actual en la transición a la vida adulta» ${ }^{3}$ realizada entre los años 2012-2014. La información cualitativa nos permite, en este caso, observar los discursos sobre las expectativas vitales de estas jóvenes. Con las entrevistas, pretendemos recoger «el proceso significativo estructurante de la visión subjetiva de un comportamiento objetivo» (Ortí, 1986: 175) y esta dotación de significados sociales se captará a través de la expresión oral de las personas entrevistadas. Y ello frente a un lenguaje, el de las entrevistadas, en muchos casos escasamente transparente $u$ opaco y repleto de capas, en otras. Un lenguaje que se resiste a ser interpretado (Ibáñez, 1992) y donde hemos buscado los condicionantes sociales a través de sus verbalizaciones. La investigación incluía la realización de 25 entrevistas en profundidad a mujeres y hombres con edades comprendidas entre los 16 y los 34 años de distintos perfiles, las fechas del trabajo de campo fueron entre diciembre de 2013 y mayo de 2014, y la captación se realizó 
mediante contactos personales así como a través de la técnica de bola de nieve. La guía de la entrevista contenía preguntas sobre la percepción que tenían los entrevistados y las entrevistadas sobre la situación de precariedad y de desigualdad en la que se encontraban.

La temporalidad analizada han sido los últimos diez años, de 2004 a 2014. La decisión se tomó según el criterio de inicio de la crisis (2004) y el momento actual (2014).

\section{La doble desigualdad de las jóvenes españolas: mujeres y precarias}

La proporción de la población joven respecto al total de la población española ha descendido motivado por el descenso en la fecundidad. La población joven entre 15 y 34 años representaba, en 1991, el 32,26\%, en 2001, el 30,67\% y, en 2011, el 24,99\%.

Gráfico 1: Población joven sobre el total (\%)

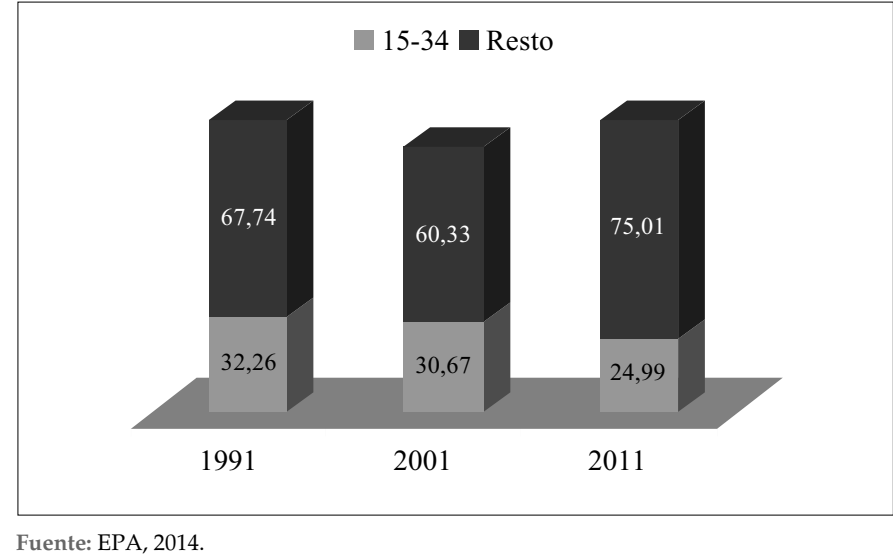

Respecto del nivel de instrucción, la población joven española supera a la media de la Unión Europea en jóvenes con estudios universitarios: un 39,2\% de los y las jóvenes españoles han completado la educación universitaria $(44,1 \%$ mujeres y $34,4 \%$ hombres), frente al $34,2 \%$ de los europeos.

La tasa de actividad de mujeres y hombres (16 a 64 años) se ha ido acortando en la última década: en 2004, era de 23 puntos porcentuales, en 2009, de 15 puntos y, en 2014, ha descendido a 10 puntos (EPA, 2015). 
Gráfico 2: Tasa Actividad por sexo y grupo de edad 2004 (\%)

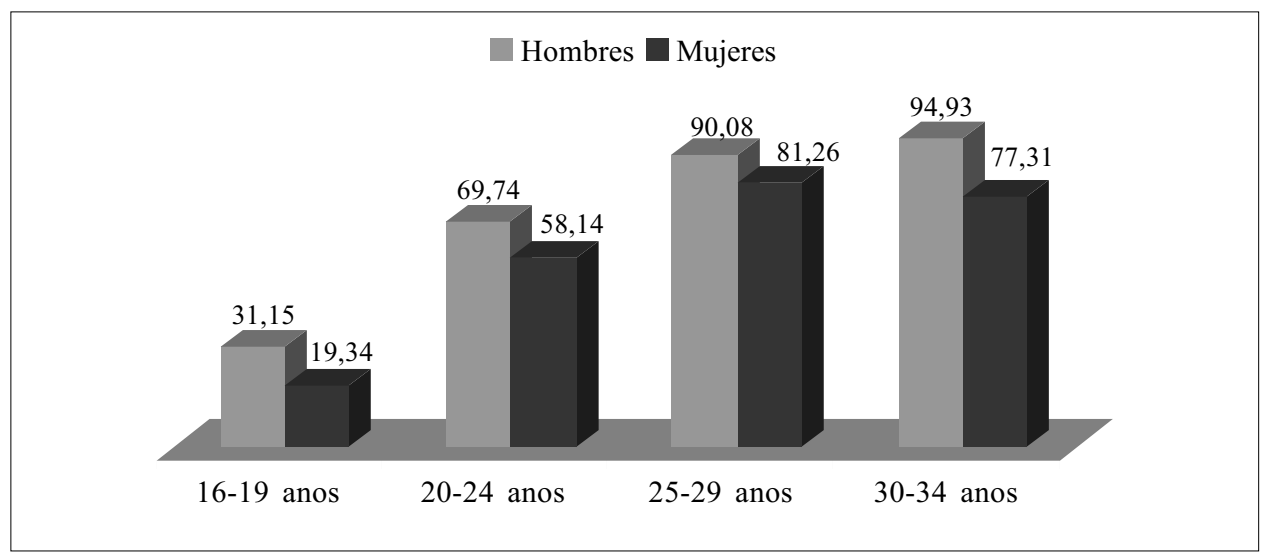

Fuente: EPA, 2014 (IV Trimestre). Elaboración propia.

Gráfico 3: Tasa Actividad por sexo y grupo de edad 2014 (\%)

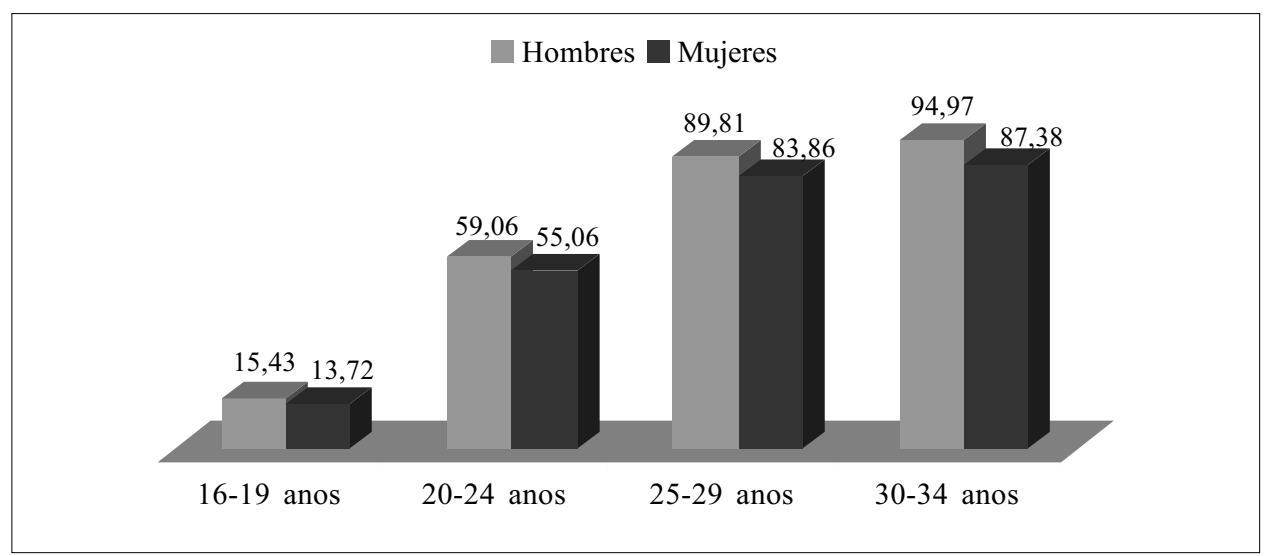

Fuente: EPA, 2014 (IV Trimestre). Elaboración propia.

\subsection{Indicadores de precariedad de las mujeres en el mercado laboral.}

La definición conceptual de precariedad alude a los siguientes aspectos sobre los que construimos unos indicadores con la finalidad de constatar la existencia de precariedad en las mujeres jóvenes en el mercado laboral.

La tasa de desempleo

Respecto a la tasa de desempleo, las siguientes gráficas muestran el progresivo incremento de la tasa de desempleo en los grupos de edad más jóvenes. 
Ahora bien, y pese a que la tasa de desempleo siempre es más alta para las mujeres, la distancia entre los sexos se acorta. Ello no quiere decir que haya disminuido la tasa de desempleo femenina sino más bien que la tasa de desempleo masculina se ha incrementado notablemente con motivo de ser los sectores masculinizados, la industria y la construcción, los más afectados por la crisis.

Gráfico 4: Tasa de desempleo por sexo y edad 2004 (\%)

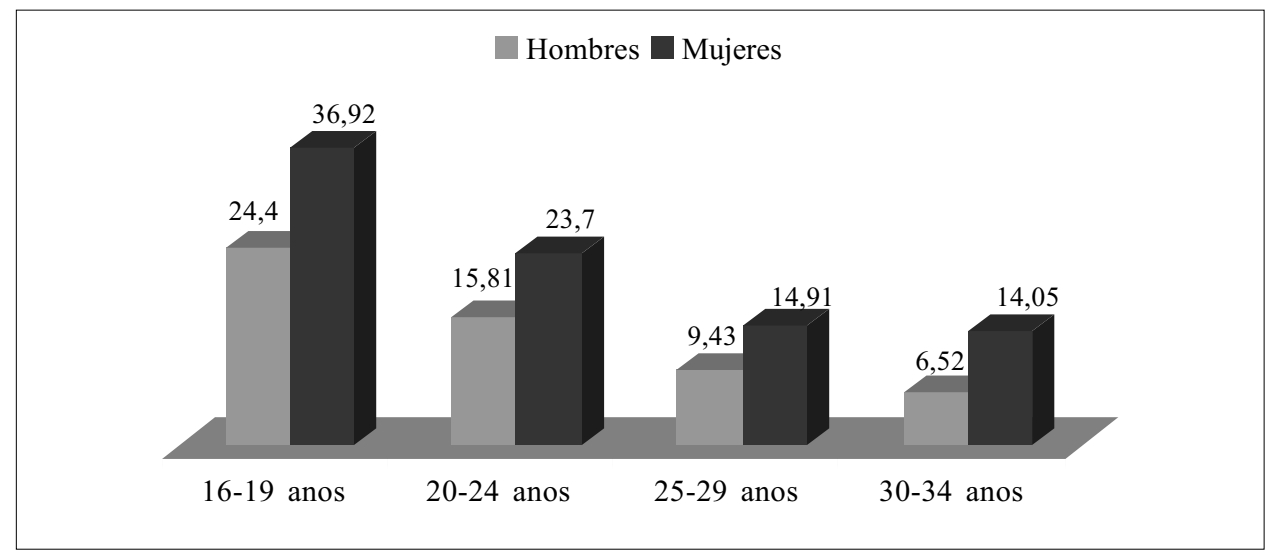

Fuente: EPA, 2014 (IV Trimestre). Elaboración propia.

Gráfico 5: Tasa de desempleo por sexo y edad 2014 (\%)

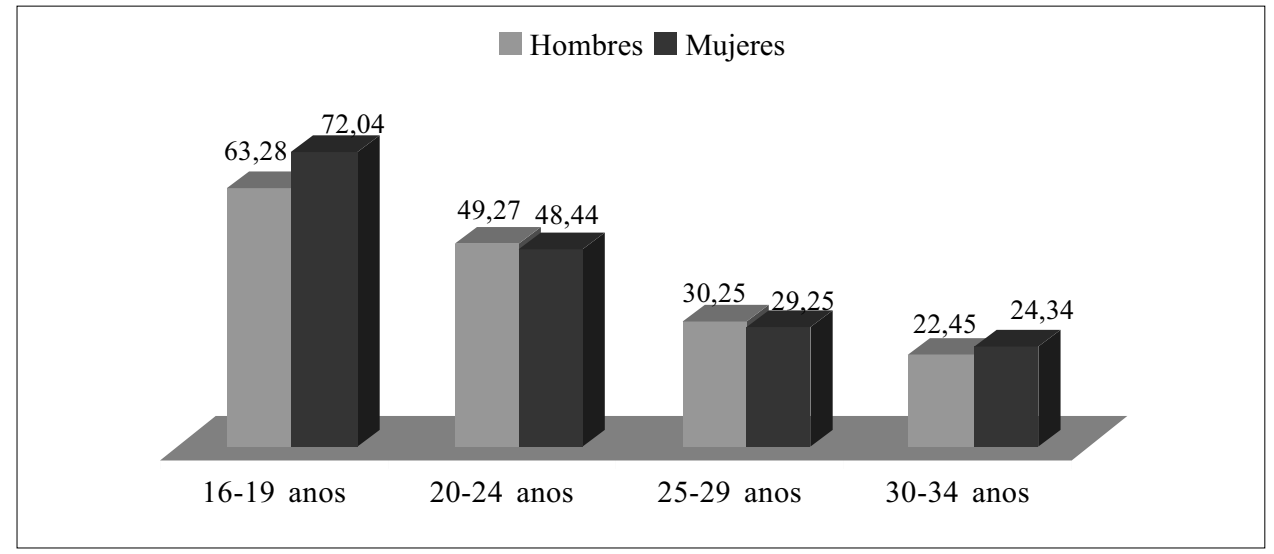

Fuente: EPA, 2014 (IV Trimestre). Elaboración propia.

Las tasas de desempleo juvenil se han incrementado en todos los países europeos desde el inicio de la crisis. La tasa de desempleo en la Unión Europea (27) de los menores de 24 años, en 2014, es de 22,6\% para los hombres y 21,3\% para las mujeres (EUROSTAT, 2015). Las tasas españolas doblan a las tasas europeas. 
Las altísimas tasas de desempleo en España traen consigo una mayor precariedad (Otaegui, 2014) que produce una reducción de los salarios, una inseguridad ante el futuro y una continuidad en el mercado laboral que degrada la calidad de los empleos impidiéndoles tener un empleo estable y duradero, no sólo en cuanto al contrato laboral, sino en cuanto al contenido desarrollado en el puesto de trabajo (Sennett, 2001).

La mayor vulnerabilidad de las mujeres ante el mercado laboral conlleva un riesgo a ocupar puestos de trabajo con posibilidad de pasar, posteriormente, al desempleo debido a la segregación laboral femenina en sectores estacionales y temporales y menos cualificados, lo que permite una mayor rotación en las contrataciones.

Tipos de jornada laboral. ¿Jornadas femeninas y jornadas masculinas?

La jornada a tiempo parcial se ha ido incorporando progresivamente en el ordenamiento jurídico laboral español justificada como una manera de facilitar la conciliación de la vida laboral y familiar, sobre todo de las mujeres, y ocultando la precariedad en la que se sitúa el trabajador o trabajadora que realiza este tipo de jornada, en relación a su menor salario, a sus menores posibilidades de promocionar, pérdida de derechos sociales y, consecuentemente, menor jubilación futura. La jornada a tiempo parcial manifesta la ruptura con el modelo fordista de jornada de trabajo continúa, semanal y para toda la vida, actualmente cuestionada por el modelo de flexibilidad neo-liberal (Alonso, 2000; Miguélez, 2004; Prieto, Ramos y Callejo, 2008).

En España, el trabajo con jornada a tiempo parcial se ha ido incrementando en los últimos diez años para ambos sexos, pero especialmente para las mujeres. En 2004, el 17,3\% del total de las mujeres ocupadas lo hacía a tiempo parcial, mientras que en 2014, la proporción ascendió a 26,1\%.

Tabla 1

Población joven según tipo de jornada (\%)

\begin{tabular}{lcccccc}
\hline & \multicolumn{2}{c}{ 2004 } & \multicolumn{2}{c}{ 2009 } & \multicolumn{2}{c}{ 2014 } \\
\cline { 2 - 7 } & T. Completo & T. Parcial & T. Completo & T. Parcial & T. Completo & T. Parcial \\
\hline Hombres & & & & & & \\
16-19 años & 88,6 & 11,4 & 67,4 & 32,6 & 50,0 & 50,0 \\
20-24 años & 91,4 & 8,6 & 81,9 & 18,1 & 70,8 & 29,2 \\
25-29 años & 95,1 & 4,9 & 91,0 & 9,0 & 83,2 & 16,8 \\
30-34 años & 98,0 & 2,0 & 95,3 & 4,7 & 90,9 & 9,1 \\
\hline Mujeres & & & & & & \\
16-19 años & 68,3 & 31,7 & 44,1 & 55,9 & 30,5 & 69,5 \\
20-24 años & 79,0 & 21,0 & 65,1 & 34,9 & 53,3 & 46,7 \\
25-29 años & 84,6 & 15,4 & 80.9 & 19,1 & 67,5 & 32,5 \\
30-34 años & 84,3 & 15,7 & 80,0 & 20,0 & 76,9 & 23,1 \\
\hline
\end{tabular}

Fuente: EPA 2004, 2009 y 2014 (IV Trimestre). Elaboración propia 
La Encuesta de Población Activa (EPA) pregunta por los motivos de ocupar dicha jornada laboral. Las respuestas son las siguientes: formación; «cuidado de niños o de adultos enfermos incapacitados o mayores»; obligaciones familiares o personales; «no haber podido encontrar trabajo de jornada completa»; «no querer trabajo de jornada completa» $u$ otros motivos.

El motivo referente al «cuidado de niños o de adultos enfermos, incapacitados o mayores», se relaciona directamente con el rol de cuidado. En la EPA 2014 (INE, 2015), sobre un total de 260.400 respuestas que aludían a este motivo, el $96,2 \%$ eran mujeres y entre los grupos de edad, los comprendidos entre los 30 y los 44 años son los más frecuentes.

Ahora bien, no todas las mujeres que ocupan puestos de trabajo a tiempo parcial aluden a las obligaciones familiares. El motivo de «no haber podido encontrar trabajo de jornada completa» resulta bastante más citado, sobre todo por parte de las mujeres, explicando los cambios que se están produciendo en las condiciones laborales del actual mercado laboral flexible (Plantenga et al, 2013). Si en 2009, eran 1.136,4 personas (el 75,7\% de ellas mujeres), en 2014 esta cantidad había ascendido hasta 1.769,1 ( $71,7 \%$ mujeres). Por grupos de edad, se observa un incremento progresivo en los grupos entre 16 y 29 años y a partir de esta edad se inicia un descenso en la proporción de personas que aluden a este motivo.

\section{Trabajo continuo o temporal}

El empleo temporal es un indicador obvio de precariedad laboral debido a que impide al trabajador o trabajadora, no sólo tener una seguridad económica continua sino, como señala Pierre Bourdieu (1999), carecer de un futuro con estabilidad y seguridad debido a las escasas posibilidades de promocionar, a la pérdida de derechos sociales y a una menor pensión de jubilación para la vejez.

Tabla 2

Trabajadores/as temporales sobre el total de ocupados/as en España(\%)

\begin{tabular}{lccc}
\hline & $\mathbf{2 0 0 4}$ & $\mathbf{2 0 0 9}$ & $\mathbf{2 0 1 4}$ \\
\hline Total 15-19 años & 80,0 & 73,5 & 85,1 \\
Hombres & $\mathbf{7 9 , 3}$ & $\mathbf{7 4 , 1}$ & 83,7 \\
Mujeres & 81,3 & $\mathbf{7 2 , 8}$ & 87,2 \\
\hline Total 15-29 años & 54,6 & 44,3 & 51,9 \\
Hombres & 55,2 & 43,6 & 51,9 \\
Mujeres & 53,8 & 45,0 & 51,9 \\
\hline Total 15-64 años & 33,4 & 25,3 & 24,0 \\
Hombres & 31,8 & 23,6 & 23,6 \\
Mujeres & 35,6 & 27,2 & 24,6 \\
\hline
\end{tabular}

Fuente: EUROSTAT. 
La tabla muestra que la tasa de temporalidad para la población joven entre 15-29 años dobla a la total de la población en edad de trabajar, en España, pero lo más sorprendente es el alto porcentaje de trabajo temporal en la población comprendida entre los 15 y los 19 años. Si bien en casi todos los casos, la tasa de temporalidad femenina es ligeramente superior a la masculina, en este caso no se puede concluir que se produzcan diferencias notables entre los sexos. Señala Amaia Otaegui (2014) que el descenso de la temporalidad es debido, en mayor medida, a la desaparición de los empleos temporales más que a que se produzca un incremento de los contratos indefinidos.

Cobrar menos por ser mujer: la brecha salarial

El término «brecha salarial de género» alude a la diferencia entre los salarios percibidos por trabajadores/as de ambos sexos, calculada sobre la base de la diferencia media entre los ingresos brutos por hora del total de población trabajadora (European Commission, 2014) ${ }^{4}$.

Tabla 3

Ganancia media anual por sexo (en miles de euros)

\begin{tabular}{lccc}
\hline & $\mathbf{2 0 0 5}$ & $\mathbf{2 0 0 9}$ & $\mathbf{2 0 1 2}$ \\
\hline Total & $18.676,9$ & $22.511,47$ & $22.726,44$ \\
Hombres & $21.093,9$ & $25.479,74$ & $25.682,05$ \\
Mujeres & $15.294,8$ & $19.735,22$ & $19.537,33$ \\
\hline
\end{tabular}

Fuente: Encuesta de Estructura Salarial.

La diferencia entre los salarios de mujeres y hombres se debe, en primer lugar, a la elección de empleos, determinados previamente por la formación recibida en la educación secundaria o en la universidad y que conduce a una mayor presencia de las mujeres en sectores feminizados relacionados con servicios personales y de cuidado, educación, sanidad, servicios sociales, etc.. En segundo lugar, las mujeres ocupan puestos de trabajo con jornadas más precarias - a tiempo parcial, temporales o informales - por lo que su salario se ve disminuido. En tercer lugar, se produce también una segregación vertical en el mercado laboral que perjudica seriamente a las mujeres ya que ocupan menos puestos de dirección. En cuarto lugar, las explicaciones anteriores se complementan con la división sexual del trabajo.

4 La demanda de un salario igual por similar trabajo realizado, se incluyó en la normativa europea desde 1957, fecha de aprobación del Tratado de Roma Art 3.Punto 2. En todas las actividades contempladas en el presente artículo, la Comunidad se fijará el objetivo de eliminar las desigualdades entre el hombre y la mujer y promover su igualdad. 
Gráfico 6: Ganancia media anual por sexo y edad en 2009

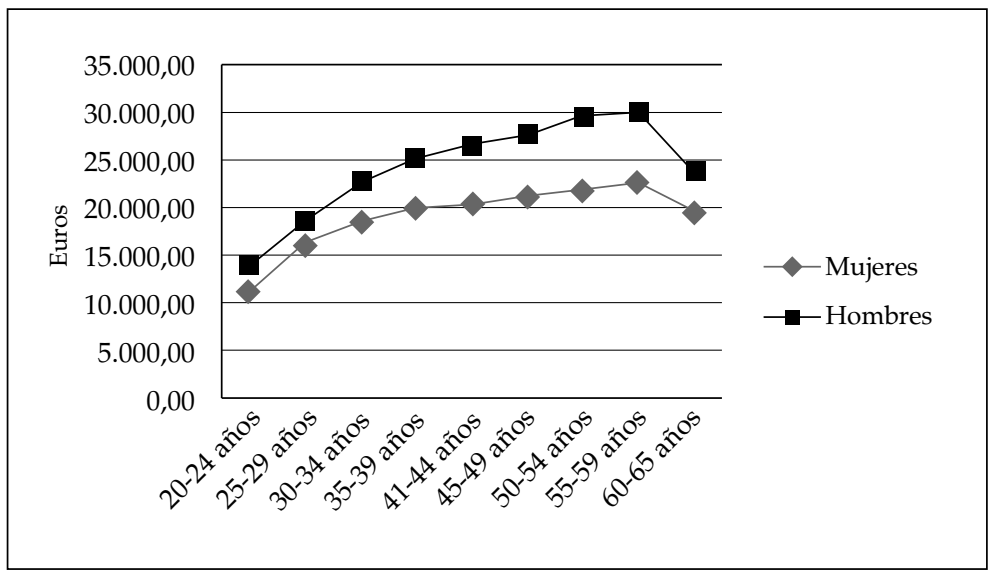

Fuente: Encuesta de Estructura Salarial 2009. INE. Elaboración propia.

Gráfico 7: Ganancia media anual por sexo y edad en 2012

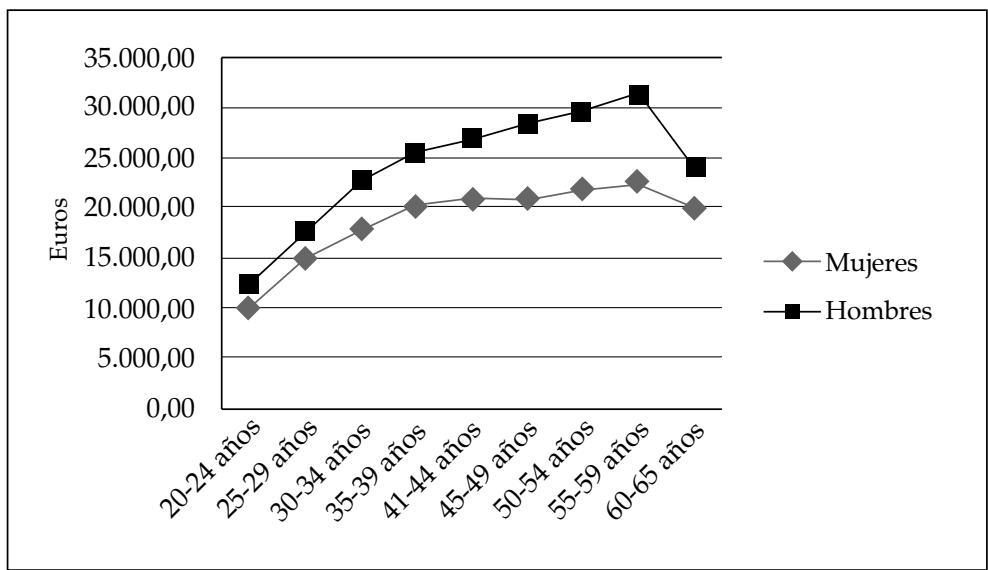

Fuente: Encuesta de Estructura Salarial 2009. INE. Elaboración propia.

Los salarios son más similares entre los sexos en los grupos jóvenes, iniciándose una divergencia a partir de los 30 años, que sólo vuelve a acercarse con la edad de la jubilación. La situación de mayor precariedad de las mujeres se corrobora al contemplar, en los dos años analizados, que se produce un incremento en

5 Desde 1978, fecha de la aprobación de la Constitución Española democrática. 
la brecha salarial en lo que respecta a las ganancias entre mujeres y hombres, entre 2009 y 2012.

\subsection{Indicadores de desigualdad de género}

A pesar de los esfuerzos realizados por parte del estado ${ }^{5}$, reflejados en la aprobación de leyes e implementación de políticas de igualdad, la igualdad entre mujeres y hombres dista mucho de haberse conseguido. Mientras las mujeres sí que se han incorporado al rol público, los hombres no han realizado en la misma proporción su aterrizaje en el rol de cuidados. De ahí que se haya producido el fenómeno de la «doble jornada» (Friedan, 1983), o «doble presencia» (Balbo, 1994), suponiendo para las mujeres una sobrecarga importante. Esta realización de doble trabajo, tiene, sin duda alguna, consecuencias en la manera en que las mujeres se incorporan al mercado laboral. Por ello, su situación de precariedad se realza al estar en el mercado laboral pero también en el ámbito doméstico, situación que no hace más que reafirmar que la igualdad no se ha alcanzado todavía.

Excedencias por cuidados

La Ley 39/1999, de conciliación de la vida laboral y familiar de las personas trabajadoras, y la Ley 3/2007 de Igualdad entre mujeres y hombres contemplan, en su articulado, la posibilidad de abandono temporal del puesto de trabajo por cuidado de personas dependientes, bien sean niños/niñas o personas enfermas o ancianas.

Tabla 4

Excedencias por cuidados

\begin{tabular}{lccc}
\hline & $\mathbf{2 0 0 5}$ & $\mathbf{2 0 0 9}$ & $\mathbf{2 0 1 2}$ \\
\hline $\begin{array}{l}\text { Por cuidado de hijos/as } \\
\text { \% Mujeres }\end{array}$ & 96,67 & 95,90 & 94,50 \\
\hline $\begin{array}{l}\text { Por cuidado de familiares } \\
\text { \%ujeres }\end{array}$ & 84,47 & 85,51 & 85,22 \\
\hline
\end{tabular}

Fuente: Mujeres en cifras. Instituto de la Mujer. Elaboración propia.

Son las mujeres las que toman mayoritariamente la excedencia aunque la ley posibilite que puedan ser ambos cónyuges los que pueden utilizarla. El hecho de que sea así responde a distintas lógicas, las mismas que llevan a que sean también las mujeres las que se decanten por solicitar la reducción de jornada por temas de cuidado (Izquierdo, 2013).

Los estereotipos construidos en torno al hombre/proveedor y la mujer/ cuidadora siguen estando presentes y presionan para que, tanto en la vida pri- 
vada como en la pública, se sigan cumpliendo salvo pena de ser considerado/a «diferente».

\section{Usos del tiempo}

Los estudios sobre usos del tiempo tienen una larga tradición en la investigación sociológica y se han convertido en una variable explicativa importante para comprender comportamientos sociales y sus diferencias entre distintos colectivos (Durán y Rogero, 2009; Fuente, 2007).

Tabla 5

Usos del tiempo por sexo

\begin{tabular}{lcccc}
\hline & \multicolumn{2}{c}{ Hombres } & \multicolumn{2}{c}{ Mujeres } \\
\cline { 2 - 5 } & $\mathbf{2 0 0 3}$ & $\mathbf{2 0 1 0}$ & $\mathbf{2 0 0 3}$ & $\mathbf{2 0 1 0}$ \\
\hline Cuidados personales & $11 \mathrm{~h} 24^{\prime}$ & $11 \mathrm{~h} 33^{\prime}$ & $11 \mathrm{~h} 21^{\prime}$ & $11 \mathrm{~h} 26^{\prime}$ \\
Trabajo doméstico y de cuidado & $1 \mathrm{~h} 30^{\prime}$ & $1 \mathrm{~h} 54^{\prime}$ & $4 \mathrm{~h} 24^{\prime}$ & $4 \mathrm{~h} 7^{\prime}$ \\
Estudios & $0 \mathrm{~h} 42^{\prime}$ & $0 \mathrm{~h} 39^{\prime}$ & $0 \mathrm{~h} 43^{\prime}$ & $0 \mathrm{~h} 39^{\prime}$ \\
Trabajo remunerado & $4 \mathrm{~h} 4^{\prime}$ & $3 \mathrm{~h} 25^{\prime}$ & $1 \mathrm{~h} 57^{\prime}$ & $2 \mathrm{~h} 9^{\prime}$ \\
Tiempo libre & $5 \mathrm{~h} 20^{\prime}$ & $5 \mathrm{~h} 23^{\prime}$ & $4 \mathrm{~h} 26^{\prime}$ & $4 \mathrm{~h} 32^{\prime}$ \\
\hline
\end{tabular}

Fuente: Mujeres en cifras. Instituto de la Mujer. Elaboración propia.

El rol de hombre proveedor y el de mujer cuidadora, tantas veces señalados a lo largo del texto, se confirma una vez más: los hombres dedican el doble de tiempo al trabajo remunerado y las mujeres, a la inversa, dedican el doble de tiempo a las tareas del hogar y de cuidado. Si bien, y tal como se ha ido apuntando, los datos de la encuesta manifiestan ligeros cambios en los comportamientos, reflejo de las transformaciones que se están produciendo en la división sexual del trabajo.

El reparto diario en el uso del tiempo entre mujeres y hombres contrasta con las opiniones dadas por la población encuestada a la pregunta sobre cuál es su forma ideal de familia: el 68,8\% responde que aquella en la que ambos miembros de la pareja se reparten el trabajo doméstico y el trabajo remunerado (CIS, 2011). La población joven, entre 18-34 años, supera en diez puntos dicha respuesta, por lo que, a nivel ideal por lo menos, su concepción de lo que debería ser la distribución del tiempo se presenta más igualitaria.

El tiempo libre también es desigualitario, registrando una evidente disposición de este tiempo diario para su uso personal e individual, fuera de los trabajos de producción y reproducción.

\subsection{Percepción de la desigualdad}

Tal y como describen una serie de indicadores con los cuales se ha pretendido dar cuenta de la situación de las transiciones a la vida adulta de las jóvenes 
españolas, la desigualdad para ellas en este período vital queda contrastada. En el análisis de entrevistas que sigue, observamos cómo las jóvenes perciben las situaciones de desigualdad muy ligadas a los estudios y a los grados de emancipación y de formación de una familia. De este modo, la fase de estudios, sobre todo la ocasión de cursar estudios superiores, es vista como una oportunidad que contagia de igualdad otras representaciones. Más si cabe cuando la enuncian en contraste con el discurso de sus progenitores/as sobre la resignación vivida en épocas anteriores.

La entrada masiva en la universidad, junto a la apertura en el hogar en la elección de estudios va forjando esa imagen de igualdad para los y las jóvenes.

creo que ahora tenemos más posibilidades, más oportunidades para poder elegir lo que queremos, [...] porque en la época de mis padres pues, también era digamos una época difícil, bueno, después de la dictadura y todo eso, [...] tuvieron que digamos resignarse más a otro tipo de vida [E16: 26 años, Lda. Publicidad, trabaja y vive en casa]

La experiencia de la generación de sus padres y madres y el énfasis en las oportunidades educativas que a las y los jóvenes se les han ido brindado en los últimos años, calan como un regalo (Martí, Alcañiz y Querol, 2015) fruto de los esfuerzos de generaciones anteriores. Muchas de estas jóvenes han experimentado, en ciertos ámbitos de su vida, una libertad biográfica sin precedentes y la posibilidad de escoger entre estudiar o no, por un lado. Además, por otro lado, la falta de presión en la decisión de optar por unos u otros estudios ayuda a seguir destacando la posibilidad de elegir en función de los gustos y aptitudes de la persona.

has estudiado tal carrera por ser chica o has hecho tal cosas en la vida por ser mujer, creo que para nada, que no ha influenciado en mi caso ni en mi casa, ni en mis hermanas ni nada. [E23: 33 años, Lda. Políticas, trabaja fuera de España]

En este sentido, el mecanismo de generar aquí un alto grado de libertad de elección (Alonso y Fernández, 2013) provoca, a su vez, la idea de que las oportunidades son iguales para ellas que para ellos. La potencia de la actual recesión ha permitido diluir la percepción de un sexo socialmente más vulnerable. La percepción del fenómeno, en consecuencia, acaba resultando la visión de una profesión vinculada a la edad, un oficio - el hecho de ser precarios - propio de los/las jóvenes.

Hoy en día yo creo que estamos un poco, en profesiones de jóvenes de gente de mi edad que está buscando así para trabajos de fines de semana y demás, no. Creo que hay igualdad. [E4: 18 años, NI-NI]

Esta virtud de escoger un futuro a través de los estudios, de autorrealizarse a través de una profesión o de una carrera laboral vitalmente estimulante, se da 
de bruces con un mercado laboral incapaz de aprovechar las capacidades de sus titulados/as (Aparici, 2004). Esta precariedad acaba cerrando, de golpe en muchos casos y en un contraste todavía más sangrante en relación a los datos expuestos más arriba, las oportunidades abiertas en la elección de estudios.

hice un máster en Microbiología, después de haberme ido al extranjero a hacer prácticas de ERASMUS. Volver aquí y intentas buscar un trabajo acorde a los estudios que tú has realizado, pero es prácticamente imposible [...] pues de momento intento trabajar en lo que sea [risa]. [E15: 27, Máster Microbiología, parada y vive en casa]

terminé la diplomatura de Turismo y entré en una agencia de viajes. Que ahí las salidas son o trabajar en hotel, o trabajar en agencias de viajes o hostelería. [...] y me quedé en hostelería, en Telepizza. [E20: 31 años, Grado Turismo, trabaja y vive en pareja]

De forma ideal, se construyen un trabajo modélico en el que se conjuguen las aspiraciones de realización familiar junto a las de una carrera profesional. Curiosamente, ese constructo ideal no queda muy cerca de nuestro país.

un trabajo bueno [...] en el que vemos que podemos cuidar bien a nuestra hija, pese a tener ese trabajo, porque tampoco se trata de tener un trabajo bueno y estar un montón de horas y tener a la niña aparcada, prefiero cobrar menos y estar con ella, entonces si él, si encontráramos la situación en Eslovaquia, la República Checa o en Ecuador, pues nos iríamos. [E18: 28 años, Lda. Música, trabaja, casada con hijos]

No obstante, esta concepción de las más jóvenes sobre una situación de igualdad en la precariedad se va modulando a medida que la transición a la vida adulta queda condicionada por la llegada de hijos/as o por un horizonte cercano con la posibilidad de tenerlos/as. Este hecho marca un nuevo sentido en sus discursos, y de un modo que parece correlacionar mejor los datos objetivos presentados anteriormente con una subjetividad que, en ciertas áreas de la vida cotidiana, descubre y desvela un mercado laboral nada conciliador con una vida más equilibrada con las necesidades familiares. Cabe destacar que estos discursos muestran cierta debilidad en tanto que se vinculan muy estrechamente a la percepción de necesidades concretas de conciliación. La investigación refleja la escasez de discursos ciertamente articulados en los que las jóvenes relacionen situaciones de desigualdad incardinadas en el mercado laboral en su amplitud y con sus variados efectos en la vida doméstica, en las relaciones de pareja, en la discontinuidad de sus carreras profesionales o, en suma, la acumulación de barreras que no permite romper con el fenómeno del 'techo de cristal'. Por el contrario, se circunscriben a las situaciones de inmediatez, en el momento en que «tengo que ir a trabajar y mi hija está enferma necesito que alguien se quede con ella. No 
tengo margen para pagar eso, entonces...» [E18: 28 años, Lda. Música, trabaja, casada con hijos]

Además, se asume y naturaliza la existencia de una conciliación del trabajo y la familiar que no atañe a las políticas públicas. Ante situaciones precarias, los costes del cuidado de las hijas y hijos terminan siendo privados. Cuando ello no es posible o deseable, los efectos sobre la desigualdad para las madres que siguen asumiendo mayoritariamente el rol del cuidado son evidentes. La disyuntiva entre pagar por que cuiden a tu hija o reducir el número de horas reproduce y alimenta un círculo vicioso de temporalidad y precariedad, casi siempre adscrito a las mujeres.

En esta línea, encontramos enunciaciones sobre el panorama de aquellas jóvenes que son madres y cuyas situaciones laborales discurren en la precariedad. De nuevo, emergen desde la descripción más concreta, al tiempo que se acaba dando justificación a la situación por el mero hecho de tener hijos.

las madres... Pues algunas en el paro, otras en la azulejera de toda la vida, y... alguna en trabajos de estos complementarios al sueldo del marido, también por los hijos y todo eso. [E21: 34 años, estudios medios, parada, viven en pareja y con hijos]

Finalmente, el hecho de tener hijas e hijos se comporta como el causante de la inestabilidad laboral. Se asume la desigualdad de una forma natural, lejos de encontrar una percepción entre las jóvenes que articule mínimamente las situaciones que se vienen describiendo en la primera parte de este análisis. La precariedad que nos cuentan no se ve distinta para mujeres y hombres, como tampoco se denuncian las barreras a la conciliación como una falta de oportunidades en la carrera laboral de la mujer.

\section{Conclusiones}

Las mujeres jóvenes en España manifiestan una situación contradictoria entre, por una parte, una mayor cualificación educativa alcanzada, y una mayor precariedad laboral, consecuencia de la ocupación en empleos a tiempo parcial, temporales y con menor sueldo y, por otra, entre la anterior situación objetiva en su posición laboral y su percepción de que mujeres y hombres son iguales y tienen las mismas oportunidades. En definitiva, el objetivo político de la igualdad choca frontalmente con la lógica del mercado laboral neo-liberal.

La diferente, y desigual, situación de las mujeres en el mercado laboral se inicia en el sistema educativo, al producirse una segregación entre ramas y carreras masculinizadas y feminizadas en estudios secundarios y universitarios. El itinerario educativo por el que optan las mujeres, en la gran mayoría de casos, se relaciona con la enseñanza, la salud, los servicios sociales, administración y servicios personales. 
A la existencia de un sistema educativo segregado por género, se une un mercado laboral desigualitario, flexible y precario, el cual afecta más a las mujeres, y una distribución asimétrica en lo que respecta a los roles de género, siendo las mujeres las más perjudicadas por su desempeño de la «doble jornada», manifestándose con claridad meridiana cuando las jóvenes son madres, acontecimiento que altera, en muchos casos, su trayectoria laboral. Son ellas las que, pese a que la legislación sobre conciliación de la vida laboral y familiar no especifique sexo, las que en mayor medida solicitan excedencias o reducción de jornada por cuidado lo cual repercute, posteriormente, en su vida laboral actual - menor promoción, menor salario - y futura - menor jubilación.

Si bien no existe actualmente una discriminación directa en cuanto a la incorporación de las mujeres al mercado laboral, siguen persistiendo en la sociedad española mecanismos indirectos de discriminación que se inician, en primer lugar, en el sistema educativo y que se reflejan, posteriormente, en ocupaciones feminizadas y masculinizadas; en segundo lugar, la persistencia de los roles de género tradicionales obliga a las mujeres a una mayor dedicación al cuidado y a las tareas domésticas contribuyendo a una alteración en su trayectoria laboral y a una divergencia en las trayectorias entre mujeres y hombres.

En suma, encontramos una distancia significativa entre la descripción - a través de los datos presentados en la primera parte - de las condiciones de acceso, de discriminación en el sueldo, la propia ausencia de políticas efectivas de conciliación, por un lado; frente a estas percepciones sobre su situación presente y futura.

\section{Referencias}

Albert, Cecilia; Toharia, Luis; Davia, María (2008), «To find or not to find a first «significant» job», Revista de Economía aplicada 46, 37-59.

Alcañiz, Mercedes (2013), «Estrategias de conciliación y segmentación social: la doble desigualdad», Sociologia. Problemas e Práticas 73, 35-58.

Alonso, Luis Enrique (2000), Trabajo y posmodernidad. El empleo débil, Madrid, Fundamentos.

Alonso, Luis Enrique; Fernández, Carlos J.(2013), Los discursos del presente. Un análisis de los imaginarios sociales contemporáneos, Madrid, Siglo XXI.

Aparici, Artur (2004), Seguimiento de las trayectorias ocupacionales de los titulados por las universidades Jaume I de Castellón, València (E.G.) y Alacant, Castelló de la Plana, Publicacions de la Universitat Jaume I.

Asián-Chaves, Rosario; Rodríguez, Vicente (2014), «Juventud y desigualdad entre hombres y mujeres en el mercado laboral», Perspectivas económicas alternativas, Valladolid, XIV Jornadas de Economía Crítica.

Balbo, Laura (1994), «La doble presencia», in Cristina Borderías et al. (1994), Las mujeres y el trabajo. Rupturas conceptuales, Barcelona, Icari, 503-514.

Becker, Gary (1987), Tratado sobre la familia, Madrid, Alianza.

Benedicto, Jorge et al. (2014), Transitar a la intemperie: jóvenes en busca de integración, Madrid, INJUVE. 
Bourdieu, Pierre (1999), Contrafuegos, Madrid, Akal.

Bradley, Harriet; Devadason, Ranji (2008), «Fractured Transitions: Young Adults' Pathways into Contemporary Labour Market», Sociology 42, 119-136.

Brunet, Ignasi; Pizzi, Alejandro (2013), «La delimitación sociológica de la juventud», Última década 38, 11-36.

Campillo, Inés (2010), «Políticas de conciliación de la vida laboral y familiar en los regímenes de bienestar mediterráneos: los casos de Italia y España», Política y sociedad 47(1), 189-213.

Cano, Ernest (2000), Precariedad laboral, flexibilidad y desregulación, Germania, Valencia.

Casaca, Sara F.; Damião, Sónia (2011), «Gender (In)equality in the labour market and the Southern European Welfare States», in Elisabetta Addis; Paloma de Villota; Florence Degavre; John Eriksen, Gender and Well-being, Londres, Ashgate, 183-200.

Casaca, Sara F. (2012), Mudanças laborais e relações de género, Coimbra, Almedina.

Casal, Joaquin et al (2006), «Itinerarios y trayectorias. Una perspectiva de la transición de la escuela al trabajo», Trayectorias 22, 9-23.

Casal, Joaquin et al (2011), «Aportaciones teóricas y metodológicas a la sociología de la juventud», Papers 79, 21-48.

Castel, Robert (1997), La metamorfosis de la cuestión social: una crónica del salariado, Buenos Aires, Paidós.

Castel, Robert (1999), «Vulnerabilidad social, exclusión: la degradación de la condición salarial», in Jorge Carpio; Irene Novacovsky (comps,), De igual a igual. El desafío del Estado ante los nuevos problemas sociales, Buenos Aires, FCE, 25-29.

Centro de Investigaciones Sociológicas (CIS) (2007, 2008, 2009), Sondeo sobre la juventud, Madrid, CIS.

Diaz Martínez, Capitolina; Dema Moreno, Sandra (2013), Sociología y género, Madrid, Tecnos.

Durán, Ma Ángeles; Rogero, Jesús (2009), La investigación sobre los usos del tiempo, Madrid, CIS.

Encuesta de Población Activa (EPA), Instituto Nacional de Estadística, Madrid [en línea] disponible en www.ine.es [consultado en 04/05/2015].

Erikson, Eric H. (1980), Identidad, juventud y crisis, Madrid, Taurus.

Esping-Andersen, Gosta (1993), Los tres estados del Estado de bienestar, Valencia, Alfons el Magnànim.

Estefanía, Joaquin (2012), La economía del miedo, Barcelona, Círculo de Lectores.

European Commission (2014), Tackling the gender pay gap in the European Union, Luxemburgo, Direção Geral da Justiça.

Eurostat (2015), Labour Force Survey; Youth on the Move y European Quality of Life Survey [en línea] disponible en http://ec.europa.eu/eurostat [consultado en 24/07/2015].

Feixa, Carles (1998), De jóvenes, bandas y tribus, Barcelona, Ariel.

Feixa, Carles (2001), Generació \& La joventut al segle XXI, Barcelona, Observatori Catalá de la Joventut.

Feixa, Carles (2014), De la generación \& a la generación \#. La juventud en la era digital, Barcelona, Ned Ediciones.

Ferreira, Virginia; Monteiro, Rosa (2013), Trabalho, igualdade e diálogo social. Estratégias e desafios de um percurso, Lisboa, CITE.

Ferreira, Vítor; Nunes, Cátia (2010), «Les trajectoires de passage à l'âge adulte», Europe, Politiques sociales et familiales 102, 21-38.

Friedan, Betty (1983), La segunda fase, Barcelona, Plaza\&Janés.

De la Fuente, Miguel (dir.) (2007), Usos del tiempo, estereotipos, valores y actitudes, Madrid, Instituto de la Mujer. 
Furlong, Andy; Cartmel, Fred (1997), Young people and social change, Buckinghamshire, Open University Press.

Furlong, Andy (ed.) (2013), Handbook of Youth and Young Adulthood. New perspectives and agendas, Nueva York, Routledge.

Gil Calvo, Enrique (2009), Crisis crónica. La construcción social de la gran recesión, Alianza, Madrid.

Guerreiro, María das Dores; Abrantes, Pedro (2005), «Transiciones a la vida adulta en la era de la globalización, recorridos de incertidumbre», Recerca. Revista de pensament $i$ analisi 5, 63-86.

Harvey, David (2007), Breve historia del neo-liberalismo, Madrid, Akal.

Ibáñez, Jesús (1992), Más allá de la sociología: El grupo de discusión: teoría y crítica, 3ạ edición, Siglo XXI, México, D.F.

Informe Foessa Precariedad y cohesion social (2014), Madrid, Fundación Foessa, Cáritas.

Instituto de la Juventud (2013), Informe Juventud en España 2012, Madrid.

Instituto de la Mujer (2006), Las mujeres jóvenes y el trabajo, Madrid, Instituto de la Mujer, Ministerio de Trabajo y Asuntos Sociales.

Izquierdo, Ma Jesús (2013), «La socialización de género», in Capitolina Díaz e Sandra Dema, Sociología y Género, Madrid, Tecno, 65-86.

Kovacs, Ilona (2005), Flexibilidade e emprego. Riscos e oportunidades, Oeiras, Celta.

Leccardi, Carmen (2005), «Facing Uncertainty. Temporality and Biographies in the New century», Young 13(2), 123-146.

Marti, Ana; Alcañiz, Mercedes; Querol, Vicent (2015), «Free, precarious and scammed. Representations and expectations of social intergenerational mobility of Spanish youth», Comunicação apresentada no Congresso Journal of Youth Studies. Conference 2015, Copenhague.

Miguélez, Faustino (2004), «La flexibilidad laboral», Trabajo. Revista de la Asociación estatal de Centros Universitarios de Relaciones laborales y Ciencias del Trabajo 13, 17-36.

Moreno, Almudena (coord.) (2012), La transición de los jóvenes a la vida adulta. Crisis económica y emancipación tardía, Barcelona, Obra Social La Caixa.

Mujeres en cifras (2014), Instituto de la mujer, [en línea] disponible en www.inmujer.es [consultado en 24/02/2014].

Oliveira, Luisa (2011), «Formas atípicas de emprego juvenil na União Europea», Sociologia. Problemas e práticas 66, 27-48.

Ortí, Alfonso (1986), «La apertura y el enfoque cualitativo o estructural: la entrevista abierta o semi-directa y la discusión de grupo», in Manuel García Ferrando; Jesús Ibáñez; Francisco Alvira (comp.), El análisis de la realidad social. Métodos y técnicas de investigación, Madrid, Alianza, 219-281.

Otaegui, Amaia (2014), El deterioro laboral de las mujeres como efecto de la crisis, Madrid, Fundación $1^{\circ}$ de Mayo.

País, José Machado (2002), «A juventude como fase de vida: dos ritos de passagem aos ritos de impasse», Saúde e Sociedade 18(3), 371-381.

Plantenga, Janneke; Remery, Chantal; Samek, Manuel (2013), Starting fragile. Gender differences in the youth labour market, Luxemburgo, Comissão Europeia, Direção Geral da Justiça.

Prieto, Carlos; Ramos, Ramón; Callejo, Javier (coord.) (2008), Nuevos tiempos del trabajo. Entre la flexibilidad competitiva de las empresas y las relaciones de género, Madrid, CIS.

Primer Informe sobre la Desigualdad en España (2013), Madrid, Fundación Alternativas.

Scott, Jacqueline; Crompton, Rosemary; Lyonette, Claire (eds.) (2010), Gender inequalities in the $21^{\text {st }}$ century. New barriers and continuing constraints, Cheltenham, Edward Elgar. 
Sennett, Richard (2001), La corrosión del carácter, Barcelona, Anagrama.

Standing, Guy (2013), El precariado. Una nueva clase social, Barcelona, Pasado\&Presente.

Stiglitz, Joseph E. (2010), Caida libre, Madrid, Taurus.

Stiglitz, Joseph E. (2002), El malestar en la globalización, Barcelona, Círculo de Lectores.

Stratigaki, Maria (2004), «The cooptation of Gender concepts in the European Union Policies: the case of Reconciliation of work and family», Social Politics 11(1), 30-56.

Torns, Teresa; Carrasquer, Pilar (2007), «Cultura de la precariedad: conceptualización, pautas y dimensiones. Una aproximación desde la perspectiva del género», Sociedad y Utopía, Revista de Ciencias Sociales 29, 139-156.

Torns, Teresa (2011), «Las mujeres y el empleo en España. ¿Un futuro venturoso?», Retos y oportunidades para la igualdad de género, Madrid, FOREM, 29-50.

Torns, Teresa; Recio, Carolina (2012), «Las desigualdades de género en el Mercado de trabajo: entre la continuidad y la transformación», Revista de Economía Crítica 14, 178-208 .

Webb, Janette (2010), «Gender and the post-industrialism shift», in Jacqueline Scott; Rosemary Crompton; Calre Lyonette, Gender Inequalities in the $21^{\text {st }}$ century: new barriers and continuing constraints, Cheltenham, Edward Elgar, 85-108.

Yuval-Davis, Nira (2006), «Intersectionality and Feminist Politics», European Journal of Women's Studies 13(3), 193-209.

Mercedes Alcañiz. Profesora titular de sociología y Directora de la Unidad de Igualdad de la Universidad Jaume I de Castellón. España. moscardo@uji.es

Vicent Querol. Profesor de sociología en la UJI. Imparte docencia en Turismo y Publicidad. Sus áreas de interés son la juventud, ocio, turismo, el desarrollo rural o el uso de las TIC en jóvenes y mayores. vquerol@uji.es

Ana Martí. Socióloga y profesora de sociología de la Universidad Jaume I. Doctora por esta misma universidad. Principales líneas de investigación y publicaciones: la sociología del género y los estudios de juventud. marti@uji.es

Dept. Filosofia y Sociología. Facultad de Ciencias Humanas y Sociales. Av. Sos Baynat s/n. 12071 Castellón. España

Artigo recebido em 29 de março de 2015 e aceite para publicação em 15 de setembro de 2015. 
ANEXO

Perfiles de los y las entrevistadas

\begin{tabular}{|c|c|c|c|c|c|}
\hline \multicolumn{6}{|c|}{ DE 16 A 24 AÑOS } \\
\hline $\begin{array}{c}\text { Número } \\
\text { entrevista }\end{array}$ & Sexo & Edad & $\begin{array}{c}\text { Nivel } \\
\text { educativo }\end{array}$ & $\begin{array}{c}\text { Situación } \\
\text { laboral }\end{array}$ & $\begin{array}{l}\text { Situación de } \\
\text { convivencia }\end{array}$ \\
\hline 1 & M & 17 & $1^{\circ}$ Bachiller & Estudiante & Padre/Madre \\
\hline 2 & M & 22 & $4^{\circ}$ Magisterio & Estudiante & Padre \\
\hline 3 & $\mathrm{H}$ & 18 & $1^{\circ}$ Bachiller & Estudiante & Padre/Madre \\
\hline 4 & M & 18 & & NI-NI & Madre \\
\hline 5 & $\mathrm{H}$ & 23 & Estudiante graduado & NI-NI & Madre \\
\hline 6 & $\mathrm{H}$ & 24 & Modulo formativo & Parado & Tíos \\
\hline 7 & $\mathrm{H}$ & 27 & ESO & Parado & Padre/Madre \\
\hline 8 & M & 20 & $1^{o}$ Ingeniería & Estudiante & Padre/Madre \\
\hline 9 & M & 22 & $1^{\underline{0}}$ Turismo & Estudiante & Madre \\
\hline 10 & $\mathrm{H}$ & 20 & $3^{\circ}$ Periodismo & Estudiante & Padre/Madre \\
\hline 11 & M & 29 & Superiores & Parada & Padre/Madre \\
\hline 12 & $\mathrm{H}$ & 21 & Bachiller & Activo & Independiente \\
\hline \multicolumn{6}{|c|}{ DE 25 A 34 AÑOS } \\
\hline $\begin{array}{c}\text { Número } \\
\text { entrevista }\end{array}$ & Sexo & Edad & $\begin{array}{c}\text { Nivel } \\
\text { educativo }\end{array}$ & $\begin{array}{c}\text { Situación } \\
\text { laboral }\end{array}$ & $\begin{array}{l}\text { Situación de } \\
\text { convivencia }\end{array}$ \\
\hline 13 & $\mathrm{H}$ & 25 & Superiores & Parado & Padre/Madre \\
\hline 14 & $\mathrm{H}$ & 29 & Máster & Parado & Padre \\
\hline 15 & M & 27 & Máster & Parada & Padre/Madre \\
\hline 16 & M & 26 & Superiores & Activa & Padre/Madre \\
\hline 17 & M & 31 & Superiores & Activa & Independiente \\
\hline 18 & M & 28 & Superiores & Activa TP & Pareja Hijos/as \\
\hline 19 & M & 34 & Superiores & Activa TP & Pareja Hijos/as \\
\hline 20 & M & 31 & Superiores & Activa & Pareja \\
\hline 21 & M & 34 & Módulo formativo & Parada & Pareja Hijos/as \\
\hline 22 & M & 34 & Superiores & Activa (Autoempleada) & Pareja \\
\hline 23 & M & 33 & Superiores & Activa & Independiente \\
\hline 24 & $\mathrm{H}$ & 33 & Primarios & Parado & Pareja Hijos/as \\
\hline 25 & $\mathrm{M}$ & 33 & Doctorado & Activa (Autoempleada) & Independiente \\
\hline
\end{tabular}

Fuente: Mujeres en cifras. Instituto de la Mujer. Elaboración propia. 